\title{
Éditorial: \\ Réponses à facettes multiples et innovatrices aux questions complexes de la recherche sur le vieillissement
}

Comme dans la majorité des domaines de la recherche scientifique, la recherche sur le vieillissement se caractérise par plusieurs éléments fondamentaux. Ainsi, le devis de recherche doit avoir une validité interne et externe incontestable, les méthodes de mesure et de techniques d'analyse doivent convenir aux hypothèses et aux données (Mangen, 1995). Toutefois, les attentes sont de plus en plus grandes vis-à-vis de la recherche sur le vieillissement. Il est maintenant généralement reconnu que la recherche sur le vieillissement informe la prise de décisions en matière de politiques publiques et de programmes. Il est également reconnu que les questions touchant les politiques pour les aînés sont complexes et pressantes. Les facteurs ayant une incidence sur l'autonomie ou la vulnérabilité des personnes âgées comportent de multiples facettes. Le soutien de l'autonomie et les soins aux membres de la famille ne sont pas uniquement le fait des systèmes de soins de santé ou de services sociaux. Les uns et les autres se combinent aux réseaux de soutien informel encore plus cruciaux (Jeans, 1994, p. 31).

\section{Initiatives fédérales favorisant la pluralité méthodologique}

Il y a un certain nombre d'années, Santé Canada a reconnu que la création d'un milieu de recherche reflétant cette complexité devait être favorisée. Par conséquent, il a établi le programme de recherche sur l'autonomie des aîné-e-s (PRAA). L'objectif du programme consiste à promouvoir les initiatives nationales de recherche mettant un accent égal sur les déterminants de l'autonomie au plan social, économique et de la santé des personnes âgées d'aujourd'hui et de demain et ce, selon une perspective individuelle ou collective. À cette fin, le PRAA a lancé une initiative de financement appelée "programmes de recherche appliquée». Ce projet fédéral a pour objectif de "réunir des équipes de recherches désirant élargir notre compréhension de ces questions" (Jeans, 1994, p. 31).

En avril 1994, le conseil consultatif du PRAA est allé de l'avant en organisant un symposium ayant pour thème "Diversité et qualité méthodologiques de la recherche en santé et de la recherche sociale appliquées dans le domaine du vieillissement». La raison d'être du symposium était de discuter de la diversité des méthodologies de recherche appliquée, de leur pertinence et de leurs points forts respectifs en vue de l'approfondissement des connaissances dans le domaine du vieillissement.

Le symposium a réuni plus de 100 chercheurs et décideurs canadiens nou- 
veaux venus ou chevronnés oeuvrant dans le secteur du vieillissement. Le programme et le format axés sur l'interaction ont suscité beaucoup d'enthousiasme parmi les participants. Ce numéro spécial est le fruit des efforts et de la volonté de bon nombre de ces participants qui désiraient étendre la discussion sur la pluralité méthodologique à un plus vaste public. Même si le PRAA a produit un compte rendu du symposium (SIRP, 1994), de nombreux conférenciers ont soumis une version modifiée de leur communication pour publication éventuelle dans ce numéro spécial de La Revue canadienne du vieillissement.

\section{Les objectifs de la pluralité méthodologique}

L'un des objectifs de ce numéro spécial consiste à fournir aux chercheurs et éducateurs, aux décideurs ainsi qu'aux représentants d'organismes pour les aînés canadiens un instrument pour mieux comprendre et connaître les méthodologies de recherche. Les articles publiés dans ce numéro mettent en lumière certaines méthodes de recherche à facettes multiples et innovatrices ayant faites leur preuve dans le domaine du vieillissement. Plus précisément, cette publication vise à faire avancer la recherche sur le vieillissement au Canada en sensibilisant ses lecteurs à l'existence et à l'utilisation de vastes banques de données, de méthodes qualitatives et quantitatives, de techniques multi-méthodes qui combinent les méthodes de recherche qualitative et quantitative, les méthodes de recherche action, les méthodes utilisées dans les stratégies de promotion de la santé, les méthodes de recherche féministe, ainsi que l'utilisation d'études longitudinales. De plus, les objectifs de ce numéro spécial, comme ceux du symposium, consistent à renseigner davantage et susciter l'intérêt envers la conception de méthodes multidisciplinaires, de collaboration et de participation dans le cadre de recherches portant sur les personnes âgées et les questions ayant trait au vieillissement; de faire connaître davantage l'ampleur, la variété et le mérite de l'éventail des modèles de recherche; et de stimuler les initiatives et l'intérêt envers les méthodes de recherche multidisciplinaires et de collaboration.

Ces objectifs deviennent de plus en plus importants au sein des milieux de recherche où on peut observer une tendance vers un "conformisme méthodologique et statistique» (Mangen, 1995, pp. 173-174). Les chercheurs développent une loyauté inébranlable pour un cadre méthodologique particulier, et les partisans de chaque camp "soutiennent que leur méthode constitue le 'meilleur moyen' de mener des recherches...". David Mangen a récemment rejeté de telles affirmations qu'il considère "gonflées d'orgueil" et souligné que "toutes les méthodes et toutes les formes d'analyse sont requises afin d'élargir les connaissances dans le domaine du vieillissement. Une clôture prématurée n'est pas recommandée; les méthodes combinées peuvent susciter de plus grands progrès" (Mangen, 1995, p. 174). Néanmoins, la majorité des chercheurs n'utilisent qu'un type de méthodologie de recherche pendant la quasi-totalité de leur carrière. Un bon nombre des ar- 
ticles présentés dans ce numéro font appel à la combinaison de méthodes en vue d'accroître les connaissances dans le secteur du vieillissement.

Lors du symposium PRAA, la ministre de la Santé a souligné les besoins en matière de recherche de son ministère pour favoriser la "recherche multidisciplinaire fondée sur la collaboration et la participation" (Marleau, 1994, p. 21). Elle a fait remarquer que l'Étude sur la santé et le vieillissement au Canada constitue un bon exemple du genre de collaboration qui sera de plus en plus requise à l'avenir, soit une coopération entre les agences, les organismes nationaux de santé, les universités, les hôpitaux et les gouvernements provinciaux. L'étude citée en exemple a fourni des données nationales sur la prévalence de cas de démence, les facteurs de risque et les tendances en matière de prestation de soins auprès de personnes atteintes de démence.

Une des principales questions abordées dans les articles de ce numéro est la collaboration sous ses formes diverses, soit entre les chercheurs dont l'orientation et la formation proviennent de secteurs variés; entre les chercheurs, les décideurs et les prestateurs de services; et entre chacun de ces groupes et les personnes âgées.

\section{Pluralité méthodologique en matière de recherche sur le vieillissement}

L'article de Neena Chappell établit le contexte pour nombre d'autres articles publiés dans ce numéro spécial. Elle décrit dans les grandes lignes les caractéristiques du climat actuel en ce qui touche les relations entre la recherche, la politique et la pratique dans le secteur de la gérontologie à la lumière des contraintes des réalités socio-économiques. Chappell soulève la question du rôle de la recherche qualitative comme un "nouvel espoir" pour répondre à bon nombre de nos questions. Les sujets qu'elle aborde sont approfondis dans les articles de Haldemann et Lévy, et de Lefrançois, et illustrent le travail accompli par Gubrium et par Wood et Kroger. Elle souligne également que la recherche appliquée suscite plus d'attention et que les résultats de recherche seront davantage utilisés en matière d'établissement de politiques et de programmes. Pour cette raison, les questions touchant la rigueur scientifique et la possibilité de reproduction des résultats de recherche revêtiront encore plus d'importance qu'à l'heure actuelle. Finalement, elle discute des embûches et des aspects pratiques de la recherche de collaboration. Ces thèmes sont discutés plus en profondeur dans les articles de King et de McGowan et Green.

L'article de Verena Haldemann et de Ronald Lévy porte sur le bien-fondé de la recherche à méthodes multiples et sur les conditions requises pour obtenir une recherche de qualité au moyen de méthodes multiples. Dans leur article, ils discutent des contextes scientifiques et socio-politiques d'où la recherche à méthodes multiples tire son origine. L'un des principaux arguments présentés est que les analyses comparatives forment le noyau des méthodes de triangulation, et que la triangulation en elle-même exige des 
points de référence externes. Comme Haldemann et Lévy l'ont souligné, l'acquisition de nouvelles connaissances a toujours été l'objectif fondamental de la science. Ils soutiennent que la question de complémentarité entre les méthodes de recherche qualitative et quantitative est en somme une question d'objectifs scientifiques. Ils font appel à l'utilisation de modèles de construction éprouvés, même si ceux-ci ne sont pas compatibles avec les modèles positivistes, comme l'exemplifient les méthodes quantitatives.

L'article de Richard Lefrançois porte également sur la pluralité méthodologique. Il y discute d'une gamme de méthodes multiples telles que la triangulation et les méthodes mixtes. Il démontre que la pluralité méthodologique est liée au domaine de la gérontologie en pleine croissance et reflète quelque peu sa problématique. Il propose également une taxinomie de stratégies à méthodes multiples. Tout au long de l'article, le sujet est toujours lié à la nature interdisciplinaire de la gérontologie.

\section{Méthodologies qualitatives}

Lorsque le comité consultatif du PRAA, dont je suis membre, a discuté pour la première fois de la possibilité de parrainer un symposium sur la diversité méthodologique, le principal sujet de conversation portait surtout sur le rôle des méthodes qualitatives dans la recherche appliquée pour le développement de politiques. Comme Haldemann le soulignait dans son éditorial de 1993 du numéro spécial de La Revue canadienne du vieillissement sur les méthodes qualitatives, nous avons, espérons-le, passé l'étape où la crédibilité, la solidité et la rigueur de la recherche qualitative doivent être établies.

Même si les articles de Haldemann et Lévy, et de Lefrançois suggèrent divers moyens de considérer les méthodes qualitative et quantitative comme étant "complémentaires" parce qu'elles fournissent des données variées et irréductibles pouvant illustrer les différentes dimensions d'un fait observable (Haldemann, 1993, pp. 130-131), la nature fondamentalement opposée de ces méthodes de recherche est également mentionnée. "Dans le cas des chercheurs qualitatifs, les questions fermées ou les observations structurées peuvent en soi être interprétées de façon très différente par diverses personnes. Cette variation est suffisamment grande pour invalider l'ensemble de cette méthode de mesure" (Mangen, 1995, p. 151). Et, comme Murphy et Longine (1992, p. 145) l'on récemment souligné, "les faits ne se trouvent pas dans un espace absolu, non encombrés par les circonstances; les données ne sont pas pures". L'objectif principal de la recherche qualitative est l'analyse de la "signification de l'expérience de personnes, relevée dans sa forme 'vécue' au moyen d'une structure peu planifiée à l'avance" (Haldemann, 1993, pp. 130-131).

Deux articles dans ce numéro, même s'ils utilisent une méthode ouvertement "qualitative," comportent une orientation très différente. Linda Wood et Rolf Kroger démontrent comment une méthode, dans ce cas-ci une analyse du discours, peut s'appliquer à la recherche en gérontologie. Jaber Gubrium, quant à lui, situe son travail dans la gérontologie conventionnelle 
et se concentre sur une technique analytique particulière de la recherche qualitative qui démontre comment l'expérience sert dans le quotidien. Cet article discute de “l'opposition analytique» essentielle à la compréhension de la prise de parole et de la narration d'une part, et de la nature du contexte de l'expérience d'autre part. Les méthodologies conventionnelles traitent ces préoccupations comme des "problèmes de procédure" et pourtant, présentées sous l'optique de Gubrium, elles constituent les caractéristiques principales des méthodes d'observation et de narration qu'il décrit.

L'article de Gubrium aborde une question qui ne reçoit pratiquement aucune attention dans la recherche en sciences sociales, c'est-à-dire notre besoin de théories méthodologiques. Pour parvenir à cette fin, l'article de Gubrium prend la forme d'un exposé très personnel de style narratif. Il est très important d'obtenir des récits aussi détaillés du processus de recherche en vue de mieux comprendre les méthodologies "de rechange", mais on les trouve rarement dans les publications scientifiques.

Gubrium décrit non seulement la méthode qu'il recommande, mais son article en constitue un exemple. En effet, le lecteur y découvre les façons dont la recherche fonctionne comme moyen d'intervention pour un bien ou un mal. L'approche vis-à-vis de la recherche et de la méthodologie, pour laquelle Gubrium se fait le champion et qu'il démontre merveilleusement dans le cadre de ses nombreux travaux, a été adoptée par d'autres chercheurs. Les publications de Barbara Myerhoff, Number Our Days, de Jennie Keith-Ross, City of Green Benches; de Sarah H. Matthews, The Social World of Old Women; d'Arlie Russell Hochschild, The Unexpected Community; de David Unruh, Invisible Lives: Social Worlds of the Aged; et de Victor Marshall (1981) sont des exemples de ce que les chercheurs ont découvert sur le vieil âge lorsqu'ils mettent la narration et le contexte en opposition analytique dans leur propre travail.

L'article de Wood et Kroger étudie les possibilités d'un autre type de méthodologie qualitative, soit l'analyse du discours en vue de comprendre les questions touchant le phénomène du vieillissement et la vie des personnes âgées. Bien que l'objectif global de cet article consiste à mieux faire comprendre la pertinence possible de ce type de recherche parmi les gérontologues, on y trouve également une explication de l'analyse du discours par une présentation de sa raison d'être, de ses méthodes et de ses réalisations. Comme le cite un compte rendu, il s'agit d'un secteur de recherche qui "semble souvent très obscur aux chercheurs oeuvrant dans des domaines étroitement liés".

\section{Approche féministe}

De plus en plus, les chercheurs reconnaissent la pertinence des méthodes féministes dans le cadre d'études sur le vieillissement (Lopata, 1995; McDaniel, 1989). L'article de Sheila Neysmith contribue à cette discussion. Elle fixe solidement les principes et les hypothèses qui sous-tendent les méthodologies féministes en fonction de la tradition critique en sciences sociales 
et discute des différences fondamentales entre cette tradition et les écoles de pensée positiviste et interprétative et, ce faisant, se rapproche de la perspective de Haldemann et Lévy. Certains des éléments de la recherche féministe traités par Neysmith reflètent la reconnaissance croissante de "l'illusion de l'objectivité absolue" soulignée par Haldemann (1993, p. 133).

\section{Méthodologies d'action participative}

Étant donné que la recherche dépend des valeurs, il va de soi que les médecins et les personnes âgées demandent aux chercheurs de travailler avec les personnes sous étude plutôt que sur ces personnes, et de traiter avec une plus grande largeur d'esprit les questions d'éthique liées à la recherche. De plus, comme Haldemann l'a souligné dans son éditorial de 1993, il est également extrêmement important que dans le cadre de leur travail, les chercheurs utilisent le même langage que celui de leurs sujets afin d'obtenir d'eux des renseignements plus précis, et qu'ils répondent aux exigences sur le plan de l'éthique en tenant compte d'autres systèmes de valeurs que le leur. Plusieurs articles dans ce numéro portent directement sur ces préoccupations. L'article de Jean King préconise l'importance et l'utilité de la recherche action axée sur les médecins ou les sujets. D'autres élargissent les principes de la recherche action en fonction des exigences de l'étude de populations spéciales, ainsi qu'auprès des autochtones (McGowan \& Green) et des participants à des groupes d'entraide (Lavoie \& Dufort).

\section{Études longitudinales et analyses secondaires}

En majorité, les discussions sur le concept du temps en matière de recherche sur le vieillissement mettent l'accent sur les questions ayant trait à la relation entre l'âge, la période et la cohorte, et recommandent ou demandent habituellement que plus de recherches longitudinales soient entreprises ou que plus de modèles "interséquentiels» soient proposés (Mangen, 1995, pp. 154-155). Récemment, la ministre de la Santé a souligné que son ministère s'était engagé à utiliser plus efficacement les vastes banques de données et à mener des recherches longitudinales (Marleau, 1994, p. 21). Ces questions sont soulevées dans deux articles publiés dans ce numéro.

Betty Havens énumère une série de questions en matière de recherche gérontologique requérant une recherche longitudinale, et explique comment les analyses de données secondaires sont essentielles à ces enquêtes. L'article de Charlyn Black détermine et caractérise plusieurs types de vastes banques de données déjà établies et fournit des exemples de recherches liées à la santé qui reposent sur l'analyse de ces banques. Les possibilités offertes par ces données pour répondre aux questions de recherches vitales dans le domaine de la gérontologie sont bien illustrées dans le récent numéro spécial de La Revue canadienne du vieillissement $(14: 2,1995)$ ayant pour thème les questions en matière de politiques sur les soins destinés aux personnes âgées au Canada. 


\section{Recherches sur des populations spécifiques}

Les recherches menées dans des contextes cliniques ou communautaires spécifiques, ou auprès de populations spécifiques présentent des défis exceptionnels aux chercheurs. Ce numéro compte trois articles portant sur ces questions. L'article de Kenneth Rockwood discute de l'utilisation des instruments de mesure dans les soins routiniers destinés aux patients gériatriques, particulièrement si ces mesures facilitent les procédés de soins et si les résultats mesurés reflètent les résultats qui importent aux patients. La mesure des résultats dans la prestation de soins de santé auprès des personnes âgées est venu à maturité à une époque où l'assignation aléatoire des sujets est l'approche méthodologique préférée pour traiter les questions d'ordre clinique (Robertson, 1994, p. 99). L'article de Rockwood discute des nombreux défis à l'élaboration et l'adoption de tels outils de mesure, et présente l'un de ces outils servant à mesurer l'efficacité des interventions spécialisées. Francine Lavoie et Francine Dufort passent en revue des questions, des modèles et des méthodes pouvant s'appliquer à la recherche sur le fonctionnement de groupes d'entraide, et abordent les contraintes face à l'accessibilité de ces groupes à des fins de recherche en raison de leur caractéristique fondamentalement privée et anonyme. Patrick McGowan et Lawrence Green soutiennent que la promotion de la santé et la recherche participative présentent des défis et des possibilités pour étendre les bienfaits de la science aux populations n'ayant pas bénéficié des innovations offertes par les soins autonomes et l'entraide.

\section{Références}

Haldemann, V. (1993). Éditorial: Les méthodes qualitatives - pourquoi? La Revue canadiennne du vieillisement, 12(2), 117-138.

Jeans, M.E. (1994). Address. Dans Proceedings of the Symposium on Methodological Diversity and Quality in Applied Social and Health Research on Aging and Seniors' Issues (pp. 31-32). Ottawa: Seniors Independence Research Program.

Lopata, H.Z. (1995). Feminist perspectives in social gerontology. Dans R. Bleiszner \& V. Hilkevitch Bedford (Éds.), Handbook of Aging and the Family (pp. 114131). Westport, CT: Greenwood Press.

Mangen, D.J. (1995). Methods and analysis of family data. Dans R. Bleiszner \& V. Hilkevitch Bedford (Éds.), Handbook of Aging and the Family (pp. 148-177). Westport, CT: Greenwood Press.

Marleau, D. (1994). Address. Dans Proceedings of the Symposium on Methodological Diversity and Quality in Applied Social and Health Research on Aging and Seniors' Issues (pp. 20-23). Ottawa: Seniors Independence Research Program.

Marshall, V.W. (1981). Participant observation in a multiple-methods study of a retirement community: A research narrative. Mid-American Review of Sociology, 6(2), 29-43.

McDaniel, S.A. (1989). Women and Aging: A Sociological Perspective. Journal of Women and Aging, 1(1/2/3), 47-67.

Murphy, J., \& Longino, C. (1992). What is the justification approach to ageing studies? Ageing and Society, 12, 143-146. 
Programme de recherche sur l'autonomie des ainé-e-s. (PRAA). (1994). Actes du symposium sur la diversité et qualité méthodologiques de la recherche en santé et de la recherche social appliquées dans le domaine du vieillissement. Ottawa: Programme de recherche sur l'autonomie des ainé-e-s.

Robertson, A. (1994). Rapporteur: Integration of Research Methods and Outcome Measures. Dans Proceedings of the Symposium on Methodological Diversity and Quality in Applied Social and Health Research on Aging and Seniors' Issues (pp. 99-100). Ottawa: Seniors Independence Research Program.

Anne Martin-Matthews 